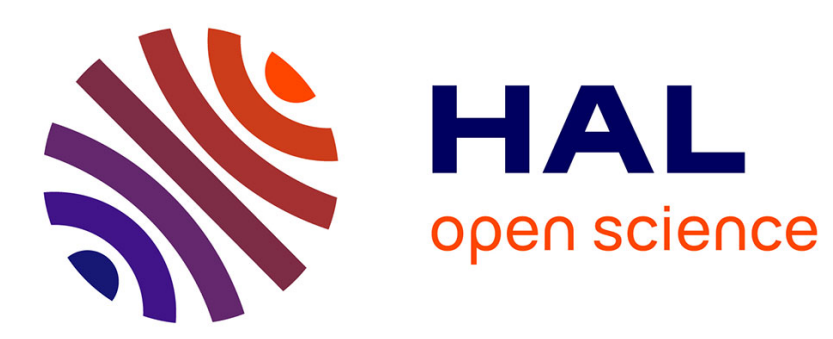

\title{
La réponse suédoise à la crise: une stratégie claire pourtant incomprise
}

Henri Bergeron, Olivier Borraz

\section{To cite this version:}

Henri Bergeron, Olivier Borraz. La réponse suédoise à la crise: une stratégie claire pourtant incomprise. Les Tribunes de la santé, 2021. hal-03499554

\section{HAL Id: hal-03499554 https://hal.science/hal-03499554}

Submitted on 21 Dec 2021

HAL is a multi-disciplinary open access archive for the deposit and dissemination of scientific research documents, whether they are published or not. The documents may come from teaching and research institutions in France or abroad, or from public or private research centers.
L'archive ouverte pluridisciplinaire HAL, est destinée au dépôt et à la diffusion de documents scientifiques de niveau recherche, publiés ou non, émanant des établissements d'enseignement et de recherche français ou étrangers, des laboratoires publics ou privés. 


\title{
La réponse suédoise à la crise : une stratégie claire pourtant incomprise
}

\author{
Henri Bergeron* \\ Olivier Borraz**
}

\begin{abstract}
Résumé
Dans ce court texte ${ }^{1}$, nous entendons montrer que la stratégie suédoise se distingue des actions menées dans d'autres pays par sa consistance: consistance historique avec les mesures décidées en amont pour se préparer à ce type de crise, là où beaucoup d'autres pays ont choisi d'improviser devant une situation jugée inédite [1]; consistance stratégique ensuite durant toute la période dans les mesures prises, en refusant de dévier de la ligne fixée malgré les pressions internes mais également externes nombreuses à s'exercer sur les autorités suédoises et notamment l'agence en charge de pilotée la crise, l'Agence de santé publique (FMH pour Folkhälsomyndigheten); là où d'autres pays ont donné au contraire l'impression d'alterner les décisions au fur et à mesure de l'évolution du virus. II ne s'agit pas pour nous de nous prononcer sur le bienfondé des mesures prises ou de leur efficacité, mais de souligner la singularité de la stratégie suédoise du point de vue de l'action collective et des politiques publiques.
\end{abstract}

\section{Mots-clés : pandémie de Covid-19, immunité collective, gestion de crise, confinement.}

Le cas suédois détonne dans le paysage global de la gestion de pandémie de Covid-19. II s'agit du seul pays dont les décisions continuent de faire l'objet d'affrontements souvent durs au plan international : entre ceux qui le citent en exemple comme un modèle (par son refus de confiner ou par l'appel à la responsabilité individuelle) et ceux qui au contraire le dépeignent comme un échec patent (en comparant ses chiffres de mortalité à ses voisins immédiats ${ }^{2}$, mais également en dénonçant une recherche d'immunité collective faisant écho au passé eugéniste de ce pays). La Suède dérange par son refus de mimer le comportement de ses voisins européens. En suggérant qu'il existe des alternatives aux solutions implantées ailleurs en Europe, elle vient casser un discours dominant qui tend à justifier les décisions prises dans un pays donné par le fait que tous les autres font de même. En défendant une approche de santé publique qui se refuse de lutter exclusivement contre une seule pathologie et le seul virus, avec le risque de produire des effets adverses de santé ailleurs,

\footnotetext{
${ }^{1}$ Ce court article s'inspire grandement des excellents travaux suivants : Jolivet $A$., Une gestion singulière de la crise sanitaire. Un système de santé décentralisé sous forte tension, 2020, Chronique internationale de I'IRES, vol. 3, n 171, p. 188-208; Et de Barrling, K. L'exception suédoise, Terra Nova, 15 janvier 2021.

${ }^{2}$ Comme le note Annie Jolivet (2020, p. 194), l'évaluation négative des effets de cette stratégie se forme à l'aune de 4 critères : "La progression rapide du nombre de cas déclarés entre la fin du mois de mars et le début du mois d'avril ; le nombre de décès rapidement très supérieur à celui des pays voisins; le nombre important de décès dans les établissements d'accueil de personnes âgées; le ratio beaucoup plus élevé entre le nombre de cas et le nombre d'habitants dans les quartiers nord de la région de Stockholm ».
} 
elle met à mal l'approche hospitalo-centrée de la crise qui domine dans la plupart des autres pays.

\section{Brève description}

Le dispositif de gestion de crise est activé dès le début du mois de février, lorsque FMH classe le virus de la Covid-19 comme un " risque important ». Un premier cas est recensé le 31 janvier et un premier décès le 11 mars. Le 16 mars, les premières restrictions sont décidées. Le choix est alors fait de ne pas recourir à un confinement généralisé, contrairement au choix fait dans d'autres pays à la même date. Cela tient notamment à l'absence de loi permettant au gouvernement de décider ce type de mesure, tout comme la fermeture des commerces, lieux publics et restaurants. Les consignes insistent d'abord sur la nécessité de s'isoler en cas de premiers symptômes en évitant d'aller tout de suite dans les centres médicaux, et de réduire les visites à l'hôpital et dans les maisons de retraite. Le gouvernement supprime également "le jour de carence pour le paiement des congés maladie afin d'inciter les personnes qui commencent à ressentir des symptômes, même bénins, à ne pas se rendre sur le lieu de travail. " [2]; et pour les congés maladie, un certificat médical n'est plus nécessaire, afin de ne pas surcharger les services médicaux. Le gouvernement interdit les rassemblements de plus de 500 personnes (mais pas dans les fêtes privées, les salles de sports, les piscines ou les bibliothèques). Les voyages à l'étranger sont déconseillés à partir du 14 mars.

Avant même que $\mathrm{FMH}$ recommande le travail à domicile, de nombreuses organisations l'autorisent pour leurs membres, tandis que l'on assiste à une chute importante d'usagers dans les transports en commun. En revanche, le gouvernement annonce le 17 mars les cours à distance pour les universités et lycées ; les crèches, écoles primaires et collèges demeurant ouverts mais susceptibles de fermeture à tout moment. Le 27 mars, le seuil des rassemblements publics est abaissé à 50 personnes; puis le 31 mars l'interdiction de rendre visite aux personnes âgées dans les maisons de retraite est annoncée.

Ces mesures renvoient à un principe de responsabilité individuelle, mais également une conviction : la meilleure manière de limiter la diffusion du virus repose sur le respect des distances (et non le port du masque ou le confinement). Le port obligatoire du masque est rejeté car accusé de créer un faux sentiment de sécurité et de conduire à ne pas respecter les autres gestes barrières. Tandis que les coûts économiques et sociaux, mais également sanitaires, du confinement relativiserait, selon $\mathrm{FMH}$, le bénéfice de cette mesure.

Cette approche est critiquée, tant en Suède par des chercheurs et médecins qui demandent publiquement des mesures plus strictes, qu'à l'étranger, car le nombre de décès qui augmente très vite est mis en regard de la situation en Norvège, Finlande et Danemark, où des mesures plus strictes seraient parvenues à contenir la pandémie. La situation est particulièrement grave dans les maisons de retraite, où se concentre un nombre très élevé de morts, et au sein de la communauté somalienne, pour laquelle les problèmes de promiscuité dans les logements et de compréhension de la langue s'ajoutent au fait que ces populations occupent souvent des emplois de service dans les maisons de retraite. Elle fait l'objet d'un premier rapport sévère rendu par la Coronakommissionen au gouvernement le 15 décembre 2020, qui pointe notamment les défaillances de la stratégie suédoise en direction des personnes âgées.

Le 10 janvier 2021, une nouvelle loi autorise le gouvernement à fermer les magasins, salles de sports, restaurants, parcs, plages et marchés: "La nouvelle loi ne permet pas au gouvernement de décider d'un confinement strict, ou d'un couvre-feu, car la constitution s'y 
oppose. Le pays est donc arrivé à la situation la plus proche d'un confinement qu'elle peut aujourd'hui organiser sans changer de constitution. » [3].

\section{Arrangements institutionnels}

Plusieurs traits marquants de cette stratégie méritent d'être soulignés.

- Tout d'abord, contrairement à de nombreux autres pays, la Suède applique les dispositifs qui avaient été définis en amont pour gérer une crise majeure. Elle ne crée pas d'organisations ad hoc mais confie le pilotage de la crise à $\mathrm{FMH}$, une agence indépendante, conformément à la loi de 2006 sur la gestion de crise et aux plans de gestion de crise qui depuis 2015 confie à cette agence la "responsabilité de la lutte contre les maladies transmissibles dans le pays et les menaces internationales à la santé publique "[2]. Elle n'adopte pas non plus de solutions inédites, comme le confinement. Ces choix peuvent surprendre mais, comme le rappelle Jolivet [4], la loi de 2006 insiste sur la continuité entre l'organisation des pouvoirs publics en temps normal et par temps de crise. Elle n'introduit donc pas de mesures d'exception, ne met pas entre parenthèses le fonctionnement des organisations ordinaires, et ne remplace pas celles-ci par des organisations temporaires. Elle repose en revanche sur une " organisation matricielle " qui croise domaine de responsabilité et niveaux territoriaux, qui organise la coordination entre des organisations qui ont I'habitude de travailler ensemble.

Certes, la loi votée en janvier 2021 donne au gouvernement des pouvoirs dont il ne disposait pas jusque-là. Mais elle ne modifie pour autant pas l'organisation des pouvoirs comme cela a pu s'observer dans d'autres pays, au nom d'une situation jugée exceptionnelle. Tant FMH que les collectivités territoriales conservent une large autonomie dans leurs décisions.

-Ensuite, "le choix de ne pas confiner totalement et de façon obligatoire l'ensemble de la population apparaît cohérent avec le système de gestion de crise suédois, très décentralisé et reposant sur un principe de responsabilité individuelle. " [5]. Là aussi, malgré la gravité de la situation, il est décidé de ne pas se départir d'une posture qui privilégie l'appel à la responsabilité à des mesures plus coercitives. Là où d'autres pays ont opté pour une recentralisation de la décision et des registres plus sécuritaires (comme en Espagne ou en Italie), la Suède opte pour une gestion de crise qui respecte les principes fondamentaux qui y gouvernent l'action publique, à commencer par la responsabilité de tous.

-Enfin, FMH défend une approche de santé publique qui refuse de sacrifier la santé de la population à des mesures centrées sur une seule pathologie. Cette posture, qui conduit à relativiser la gravité de la Covid-19 au regard d'autres risques de santé publique, est vivement critiquée au regard du nombre élevé de décès. Au moment où nous écrivons ce texte, il n'est pas certain qu'elle puisse être maintenue, tant la pression politique augmente sur le gouvernement. Mais il est remarquable que durant toute l'année 2020, elle n'ait pas été remise en cause. Cette approche a été contestée notamment parce qu'elle reposait sur la poursuite d'un objectif d'immunité collective, d'abord revendiqué par les dirigeants de FMH avant que ceux-ci nient avoir tenu de tels propos ; pourtant confirmés ensuite dans des documents écrits. Elle a également été mise à mal par des médecins hospitaliers, obligés de déprogrammer des interventions pour faire place à des patients Covid. Elle n'en demeure pas moins distinctive, au regard de la gestion hospitalo-centrée poursuivie dans la plupart des autres pays, mais également le peu d'attention accordée aux conséquences économiques, sociales et sanitaires des mesures de protection (confinement, fermetures des lieux publics et commerces, fermetures des écoles). Si le nombre de morts par millions est 
aujourd'hui très proche entre la Suède et la France, il sera important de mesurer les différences dans les années à venir sur d'autres problèmes de santé.

Janvier 2021

*Henri Bergeron est directeur de rercherche au CNRS, au Centre de Sociologie des Organisations (Sciences Po - CNRS), et coordinateur scientifique de la chaire Santé de Sciences Po.

**Olivier Borraz est directeur de recherche au CNRS et dirige actuellement le Centre de sociologie des organisations, unité mixte de recherche CNRS-Sciences Po.

Références

1- Bergeron H., Borraz O., Castel P., Dedieu F. Covid-19: une crise organisationnelle, Paris, Presses de Sciences Po, 2020.

2- Jolivet A. Une gestion singulière de la crise sanitaire. Un système de santé décentralisé sous forte tension, 2020, Chronique internationale de I'IRES, vol. 3, $\mathrm{n}^{\circ}$ 171, p. 192.

3- Barrling, K. L'exception suédoise, Terra Nova, 15 janvier 2021. p. 3.

4- Jolivet, op. cit. p. 191.

5- Jolivet, op. cit. p. 206. 